\title{
Gastrointestinal diseases during pregnancy: what does the gastroenterologist need to know?
}

\author{
Catarina Frias Gomes a , Mónica Sousa ${ }^{b}$, Inês Lourençoc, Diana Martinsc', Joana Torres ${ }^{a}$ \\ Hospital Beatriz Ângelo, Loures, Portugal
}

\begin{abstract}
Pregnancy is characterized by numerous physiological changes that may lead to a diversity of symptoms and frequently to gastrointestinal complaints, such as heartburn, nausea and vomiting, or constipation. Chronic gastrointestinal diseases require treatment maintenance during this period, raising the challenging question whether outcomes beneficial to the mother may be harmful for the fetus. In addition, certain diseases, such as acute fatty liver of pregnancy, only develop during pregnancy and may require urgent procedures, such as fetus delivery. Even though they are not present in our day-to-day practice, knowledge of pregnancy-related diseases is fundamental and collaboration between gastroenterologists and obstetricians is often necessary. Herein, we review pregnancy-related diseases and systematize the most appropriate treatment choices according to the recent literature and guidelines, so that the article can serve as a guide to the gastroenterologist regarding the medical approach to pregnancy-related gastrointestinal and liver diseases and their therapeutic management.
\end{abstract}

Keywords Pregnancy, gastrointestinal symptoms, inflammatory bowel disease, liver, endoscopy, treatment, management

Ann Gastroenterol 2018; 31 (4): 1-11

\section{Introduction}

Pregnancy is a challenging period for the gastroenterologist. Although multiple gastrointestinal complaints may occur during pregnancy (gastroesophageal reflux, constipation, etc.) most of these are mild to moderate, usually managed by the obstetrician, and therefore the gastroenterologist is seldom asked to intervene. However, certain complications of pregnancy, such as specific liver diseases, the management of pregnant women with inflammatory bowel disease (IBD), or the need for endoscopy during pregnancy, may require intervention by a gastroenterologist; therefore, is it is essential that the gastroenterologist keeps up to date with the most frequent complications and potential differential diagnoses of pregnancy (Table 1). Herein, we a comprehensive review of the

aSurgical Department, Gastroenterology Division (Catarina Frias Gomes, Joana Torres); ${ }^{b}$ Medicine Department, Internal Medicina Division (Mónica Sousa);); 'Surgical Department, Gynaecology and Obstetrics Division (Inês Lourenço, Diana Martins), Hospital Beatriz Ângelo, Loures, Portugal

Conflict of Interest: None

Correspondence to: Joana Torres, Avenida Carlos Teixeira 3, 2674-514, Loures, Portugal, e-mail: joanatorres00@gmail.com

Received 25 January 2018; accepted 26 February 2018; published online 27 April 2018

DOI: https://doi.org/10.20524/aog.2018.0264 most common gastrointestinal complications of pregnancy, providing a guide for the gastroenterologist that may be helpful in the management, diagnosis and approach to these situations.

\section{Materials and methods}

An electronic search of PubMed was conducted in order to identify relevant literature on the physiopathology, clinical presentation, treatment, maternal and fetal outcome for both gastrointestinal and liver diseases during pregnancy; this search was complemented by the most recent guidelines available (ECCO, ACG, EASL, Royal College of Obstetrics and Gynaecology).

\section{Physiological changes during pregnancy}

Pregnancy is characterized by a hemodilution state induced by volume expansion due to retention of salt and water. Consequently, there is a fall in hemoglobin and albumin concentrations. Platelet levels may decrease, but usually remain within the normal range. Alkaline phosphatase (AP) may show a 3- to 4-fold increase due to placental production. Aspartate aminotransferase, alanine aminotransferase (ALT), $\gamma$-glutamyl transferase (GGT), bilirubin, and prothrombin (PT) time remain within their normal ranges. Nevertheless, clotting 
Table 1 Synopsis of the diseases during pregnancy. Inflammatory bowel disease may be exacerbated during pregnancy. Hepatitis E is associated with a higher risk of acute fulminant hepatitis and mortality compared to non-pregnant women

\begin{tabular}{lll} 
Common GI complaints during pregnancy & $\begin{array}{l}\text { GI disoders unique to } \\
\text { pregnancy }\end{array}$ & $\begin{array}{l}\text { Diseases that may be } \\
\text { exacerbated during } \\
\text { pregnancy }\end{array}$ \\
\hline $\begin{array}{l}\text { Nausea and vomiting } \\
\text { Gastroesophageal reflux }\end{array}$ & $\begin{array}{l}\text { Hyperemesis gravidarum } \\
\text { Constipation }\end{array}$ & $\begin{array}{l}\text { Intrahepatic cholestasis of } \\
\text { pregenancy }\end{array}$ \\
& Preeclampsia/Eclampsia & Hepatitis E \\
& HELLP syndrome & Acute fatty liver of \\
pregnancy & \\
\hline
\end{tabular}

GI, gastrointestinal; HELLP, hemolysis, elevated liver enzymes and low platelet count

factor concentrations are affected by pregnancy, with a mild decrease in antithrombin III, protein $\mathrm{C}$ and protein $\mathrm{S}$, and an increase in factors I to X, XII and fibrinogen which favors a pro-coagulant state [1].

Elevated levels of hormones, such as progesterone, contribute to delayed gastric emptying. Gastric acidity is increased because of the higher production of gastrin by the placenta [2]. The fetal intrauterine growth also alters the anatomic relations between the abdominal organs: for example, the appendix may migrate upward after the $3^{\text {rd }}$ month [3].

\section{Gastrointestinal diseases}

\section{Common gastrointestinal complaints}

\section{Nausea and vomiting}

Nausea and vomiting are the most frequent medical conditions during pregnancy, affecting $50-80 \%$ of women [4]. These symptoms begin between the $4^{\text {th }}$ and $6^{\text {th }}$ week, peak at the $8^{\text {th }}$ to $12^{\text {th }}$, and often cease by the $20^{\text {th }}[4]$. Peak symptoms and drug administration often coincide with the most susceptible period to teratogenic effects. The exact mechanism of nausea and vomiting is not completely understood. It is believed that progesterone has an inhibitory effect on the smooth muscle of the pylorus and small bowel, decreasing gastrointestinal motility and delaying gastric emptying [4]. Multiple and molar pregnancies have been associated with more prevalent and severe symptoms, which can be explained by higher levels of human chorionic gonadotropin (HCG) [5]. Finally, psychological factors may also play a role. Anxiety, depression, undesired pregnancies and negative relationships with family members have been linked to a higher prevalence of nausea and vomiting [4].

The approach to nausea and vomiting entails the exclusion of other causes, especially if these symptoms persist during the second and third trimesters. Most women do not require pharmacological treatment and can be managed with alternative options and dietary modifications [6]. Diet recommendations include fractionized meals and avoidance of fatty foods and fresh vegetables that can delay gastric emptying [4]. Daily consumption of $1.5 \mathrm{~L}$ of water or drinks containing glucose, salt and potassium are recommended. Both thiamine (vitamin B1) and pyridoxine (vitamin B6) have proven their efficacy and are indicated as routine supplements in patients with protracted vomiting [4]. A 2010 Cochrane review evaluated nine randomized trials involving ginger [5]. Based on expert experience, ginger can be considered a nonpharmacologic option during pregnancy [7].

Concerning the pharmacological treatment, antiemetics can be an option for women with persistent nausea and vomiting. Histamine receptor blockers (anti-H1), such as promethazine, cyclizine, cinnarizine, doxylamine and dimenhydrinate, are considered first-line treatment, because no adverse fetal effects have been described [4]. Doxylamine has the advantage of being safe when symptoms begin (6-7 weeks). In a cohort of 608,385 pregnancies, Pasternak et al evaluated ondansetron, an $\mathrm{H} 3$ receptor blocker, and concluded that it was not associated with a significant increase in spontaneous abortion, stillbirth, major birth defects, preterm delivery or small born infants for gestational age [8]. However, there are studies documenting congenital heart defects, especially when ondansetron was taken in the first trimester. Consequently, it is recommended as second-line therapy [9]. Phenothiazines are another first-line treatment option, including prochlorperazine, chlorpromazine and perphenazine. Studies have not shown increased risk for major malformations [6]. Finally, metoclopramide, a dopamine antagonist, can also be safely prescribed. However, it crosses the placenta and is considered a second-line treatment option, given its potential extrapyramidal effects on both the fetus (third trimester) and the mother (first trimester) [9].

\section{Gastroesophageal reflux disease (GERD)}

GERD is reported by $40-85 \%$ of pregnant women, usually beginning at the end of the first trimester, and can profoundly impair the quality of life [10]. When present, GERD persists during the entire pregnancy and usually resolves after delivery. Complications such as erosive esophagitis, bleeding or strictures are rarely described [4]. For mild symptoms, lifestyle and dietary modifications are recommended [6]. Conservative measures include avoiding eating late at night, elevating the 
head of the bed by $10-15 \mathrm{~cm}$ and lying on the left side [10]. If symptoms persist, medication may be necessary. Firstline treatment is based on antacids [10]. Antacids containing calcium, and magnesium are considered safe in pregnancy. Furthermore, patients prefer to use this medication because it relieves the symptoms promptly and can be taken on demand. No teratogenic effects of these drugs have been observed in animal studies [11]. Bicarbonate-containing agents, such as sodium bicarbonate, should be avoided as they can precipitate metabolic alkalosis and fluid overload in the mother and fetus $[4,10]$. A further concern with aluminum antacids is the potential developmental retardation described when women take high doses of this medication [12]. The potential fetal toxicity when using sucralfate is associated with its aluminum component. However, no maternal or fetal adverse effects have been observed, and therefore sucralfate is usually considered safe during pregnancy [13]. Histamine type 2 antagonists are often the next-line therapy. Both cimetidine and ranitidine have been used with excellent safety profiles $[4,10]$. Although there is a safety concern with proton pump inhibitors (PPIs), in a recent meta-analysis Gill et al did not find an increased risk for major congenital malformation with PPI use during pregnancy [14]. However, some studies in the past reported dose-related embryonic and fetal mortality in animal studies with omeprazole [4]. Prospective data have shown the safety of this drug [15]. Pasternak et al, in a cohort study, did not find a significant increase of major birth defects with exposure to PPIs during the first trimester of pregnancy (odds ratio [OR] 1.10, 95\% confidence interval [CI] 0.91-1.34) [16]. In an individual analysis, there was no significant association between the use of any specific PPI and the risk of major birth defects [16]. Altogether, PPIs are nowadays considered to be safe in pregnant women and are usually reserved for GERD complications or for women who did not respond to previous treatments [13]. All agents that decrease gastric acidity should be used with caution, as they can lower iron absorption.

\section{Constipation}

Constipation usually occurs in the first and second trimester, affecting 35\% and 39\% of women, respectively. The prevalence of constipation in the third trimester and post-partum period is estimated at around $20 \%$ and $17 \%$, respectively [17]. The risk of constipation seems to be higher if documented in previous pregnancies and is associated with iron intake.

The higher prevalence of constipation in early pregnancies suggests that sex hormones are the major influence. Progesterone induces relaxation of smooth muscle, leading to hypomotility of the small and large bowel. In addition, it has been proposed that pregnant women have an increased absorption of colonic water, explained by higher levels of aldosterone induced by sexual hormones such as estrogen and progesterone. Iron intake during pregnancy can worsen constipation complaints. Finally, mechanical factors can also contribute in late pregnancy. The combined movements of the intestinal tract and uterus have been found to impede the onward movement of solid feces, obstruct defecations and initiate constipation $[18,19]$. Although a vast majority of pregnant women are found to have functional constipation, it is important to exclude mechanical causes and pay particular attention to the possibility of diabetes or hypothyroidism [18].

Non-pharmacological management of constipation includes adequate water intake and a high-fiber diet. Food volume may exacerbate the symptoms, especially in the second trimester, and fractionized meals may be an alternative $[17,18]$. Drug therapy is reserved for refractory patients because it may be associated with complications. Fiber-containing bulking agents $\left(\right.$ Metamucil $^{\oplus}$, Citrucel $^{\oplus}$ ) are probably the safest laxatives to be used in pregnancy, as they are not systemically absorbed [18]. Nevertheless, they do not relieve acute symptoms and may need several days to act. Osmotic laxatives, such as lactulose, polyethylene glycol (PEG), glycerin and sorbitol, are considered safe and animal studies have shown no teratogenic effects [18]. In fact, the American Gastroenterological Association position statement considers PEG as a lowrisk drug and the preferred drug for chronic constipation in pregnancies $[20,21]$. On the other hand, stimulant laxatives, such as sennosides and bisacodyl, should be avoided during pregnancy. Medications not recommended include mineral oil, castor oil and saline hyperosmotic agents [4,21]. Table 2 summarizes the recommended treatment options for common gastrointestinal complaints.

\section{Specific gastrointestinal diseases of pregnancy}

\section{Hyperemesis gravidarum (HG)}

HG is a severe form of nausea and vomiting associated with weight loss of more than $5 \%$ of pre-pregnant weight, dehydration and electrolyte imbalances. HG usually starts before the $22^{\text {nd }}$ week of gestation, affects $0.3-2.0 \%$ of pregnancies and sometimes requires hospital admission [22,23]. In a Canadian population-based cohort study, Fell et al reported an increased risk of HG associated with hyperthyroid disorders, psychiatric illness, previous molar pregnancy, pre-existing diabetes and asthma [24].

Currently, three major etiologies have been described in the literature. Firstly, high levels of HCG can have a stimulating effect on the secretory process in the upper gastrointestinal tract. The production of thyroid-binding globulin also increases under estrogen stimulation, leading to a decrease in free thyroxine (T4). The transient decrease in the free T4 level causes stimulation of the thyroid and the patient can develop gestational transient thyrotoxicosis, leading to vomiting. Secondly, HCG is similar to thyroid-stimulating hormone (TSH) and possibly causes hyperemesis by stimulating the TSH receptor [23]. Thirdly, there is a negative relation between the levels of prolactin and nausea/vomiting, whereas estrogens have a positive relation. Therefore, the higher levels of estrogens during pregnancy can raise the risk of HG [25].

$\mathrm{HG}$ is a diagnosis of exclusion. It is usually accompanied by hyponatremia, hypokalemia, low serum urea, raised hematocrit, metabolic hypochloremia alkalosis and ketonuria. 
Table 2 Summary of treatments of common gastrointestinal complaints. Description of pharmacological and non-pharmacological treatment for nausea and vomiting, GERD and constipation. It is also indicated whether they are first- or second-line therapy

\begin{tabular}{|c|c|}
\hline Treatments of common gastrointestinal complaints & Indication \\
\hline \multicolumn{2}{|l|}{ Nausea and vomiting treatment (including $\mathrm{HG}$ ) } \\
\hline \multicolumn{2}{|l|}{ Non-pharmacological } \\
\hline Dietary measures & All patients \\
\hline Thiamine & May be used \\
\hline Pyridoxine & May be used ${ }^{*}$ \\
\hline Ginger & May be used \\
\hline \multicolumn{2}{|l|}{ Pharmacological } \\
\hline Histamine receptor blocker (anti H1 - promethazine, cyclizine, cinnarizine, doxylamine) & $1^{\text {st }}$-line therapy \\
\hline Histamine receptor blocker (anti H3 - ondansetron) & $2^{\text {nd }}$-line therapy \\
\hline Phenothiazine (prochlorperazine, chlorpromazine, perphenazine) & $1^{\text {st }-l i n e ~ t h e r a p y ~}$ \\
\hline Dopamine antagonist (metoclopramide) & 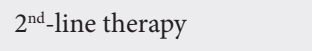 \\
\hline Corticosteroids & Non-responding patients \\
\hline \multicolumn{2}{|l|}{ GERD treatment } \\
\hline \multicolumn{2}{|l|}{ Non-pharmacological } \\
\hline Lifestyle modifications and diet & All patients \\
\hline \multicolumn{2}{|l|}{ Pharmacological } \\
\hline Anti-acids (agents containing calcium, or magnesium) & $1^{\text {st }}$-line therapy \\
\hline Sucralfate & May be used \\
\hline Histamine receptor blocker (anti $\mathrm{H} 2$ - cimetidine and ranitidine) & $1^{\text {st }}$-line therapy \\
\hline Proton pump inhibitors & When clinically indicated ${ }^{*}$ \\
\hline \multicolumn{2}{|l|}{ Constipation treatment } \\
\hline \multicolumn{2}{|l|}{ Non-pharmacological } \\
\hline Water intake and high-fiber diet & All patients \\
\hline \multicolumn{2}{|l|}{ Pharmacological } \\
\hline Fiber-containing bulking agents (Metamucil ${ }^{\circ}$, Citrucel $^{\circ}$, Perdiem $\left.^{\circ}\right)$ & $1^{\text {st }}$-line therapy \\
\hline Osmotic laxatives (lactulose, PEG, glycerin, sorbitol) & $1^{\text {st }}$-line therapy \\
\hline Sennosides and bisacodyl & Not recommended \\
\hline
\end{tabular}

GERD, gastroesophageal reflux disease; PEG, polyethylene glycol; HG, hyperemesis gravidarum; ${ }^{*}$ Not recommended according to the Royal College of Obstetricians and Gynaecologists; and ${ }^{*} G E R D$, complications and non-responding patients

Liver enzymes may be elevated in $50 \%$ of cases. Patients are dehydrated with fluid/food intolerance and weight loss due to the prolonged vomiting. Ultrasound assessment of pregnancy is mandatory, because HG may be associated with multiple and molar pregnancies [23].

No single therapy has emerged as significantly beneficial and the medical approach is based on electrolyte correction and prevention of dehydration [23]. When hospitalized, these women usually need intravenous fluid therapy to correct electrolyte disturbances. Normal saline solution is recommended to correct possible hyponatremia. As in non-severe nausea and vomiting, thiamine (vitamin B1) and pyridoxine (vitamin B6) may be appropriate to prevent severe symptoms. Thiamine also prevents Wernicke's encephalopathy in patients with prolonged vomiting. If patients fall to respond to conservative therapy, antiemetics are indicated, including histamine receptor blockers, phenothiazines and dopamine antagonists. Malnutrition may necessitate nasojejunal enteral feeding, effective in symptom control and weight gain in severe HG. Total parenteral nutrition may be used in life-threatening cases [23].

\section{Inflammatory bowel disease (IBD)}

IBD affects women during their reproductive years and it is estimated that around 50\% is diagnosed before age 32 [26]. IBD may be associated with worse pregnancy outcomes; therefore, it is recommended that the IBD physician and obstetrician follow patients closely. This is especially important since many obstetricians may not be familiar with IBD medications and may not be able to advise correctly on the maintenance of drugs during pregnancy. 
Pregnancy may influence the course of IBD. In a 10 -year follow-up cohort study, Riis et al concluded that pregnancy did not influence disease phenotype or surgery rate, but was associated with a reduced number of flares in the following years [20]. Conversely, IBD may be associated with worse outcomes during pregnancy, even during quiescent disease [26]. In a recent review of 3907 IBD patients, Cornish et al concluded that women with IBD are more likely to have preterm birth, low birth weight and caesarean section, the last one occurring especially in Crohn's disease. All these outcomes are much more frequent if the disease is active during pregnancy [27]. Indeed, disease activity is the strongest predictor of an adverse pregnancy outcome [26]. If conception occurs during quiescent disease, the risk of relapse is similar to that of a non-pregnant IBD women. Patients with ulcerative colitis have a 33\% risk of relapsing and Crohn's disease patients have a risk of around 20\% [26]. In contrast, if conception occurs during an exacerbation of disease, $2 / 3$ will have a persistent active disease and $2 / 3$ of these will deteriorate further [20].Likewise, active disease at conception is associated with a greater risk of spontaneous abortion and preterm birth, whereas disease exacerbation during pregnancy increases the risk of preterm birth, stillbirth and low birth weight [26]. In a cohort study of 462 pregnant women with IBD, Mahadevan et al also found a higher prevalence of pregnancy complications in IBD patients (OR 1.78, 95\%CI 1.13-2.81), such as preeclampsia or premature rupture of membranes [28]. Therefore, it is advised that IBD women have a 3- to 6-month period of sustained remission before pregnancy is attempted. The ongoing prospective, multicenter study "Pregnancy in Inflammatory Bowel Disease and Neonatal Outcomes" (PIANO) found a higher rate of exacerbation in ulcerative colitis patients compared to Crohn's disease patients during pregnancy [29].

Concerning the mode of delivery, the majority of patients can have a normal term delivery. Crohn's disease patients with active perianal disease or active rectal involvement should deliver by C-section; likewise, although not being an absolute indication, it is normally advised that women with J pouches deliver by $\mathrm{C}$-section to preserve fecal continence $[26,30]$.

A range of diagnostic markers may be used to assess disease activity during pregnancy, including erythrocyte sedimentation rate, hemoglobin, albumin, and C-reactive protein (CRP). However, the physiologic changes during pregnancy affect these biomarkers, especially CRP, albumin, hemoglobin, and leukocyte count. According to recent studies, fecal calprotectin (FC) concentrations are not altered in healthy pregnant women and therefore may be used to monitor disease activity during pregnancy $[26,31,32]$. In a multicenter observational study conducted in Denmark, Julsgaard et al suggested the use of FC in combination with physician global assessment to monitor disease activity during all trimesters [32]. Nevertheless, Meuwis et al did not find a statistically significant correlation between FC alone and disease activity during any of the 3 pregnancy trimesters [33]. Further research is warranted to define the role of FC during pregnancy in women with IBD, even though it has the potential to be used as biomarker of active disease. Sigmoidoscopy should only be used in severe cases, such as those with lower gastrointestinal bleeding. Abdominal pain and diarrhea are weaker indications for the procedure [26,34]. Ultrasound, used to monitor the development of the fetus, is safe, and low-dose X-rays pose a minimal risk to the fetus.

Treatment of IBD should always be considered, because active disease poses a higher risk to the fetus and the mother than does the medication [26]. In general, most treatments can be used during pregnancy, with the exception of methotrexate and thalidomide, which are teratogenic. 5-aminosalicylates are considered to be safe during pregnancy, although sulfasalazine interferes with folic acid absorption and higher doses of supplementation are needed [6]. Azathioprine crosses the placenta and has been associated with newborn anemia [6,29]. However, most studies have shown that it is a safe therapy during pregnancy. It is more reasonable to continue thioprine monotherapy to maintain IBD remission than to stop it and increase the flare risk. Evidence of cyclosporine use in IBD is limited to small series of women who had severe relapses during pregnancy [26]. In transplanted patients it was associated with low birth weight and preterm birth, although this finding was not described in IBD patients. Anti-tumor necrosis factor agents (anti-TNF) infliximab and adalimumab are considered low-risk agents. Several studies suggested that infliximab is not teratogenic and does not lead to pre-term delivery or low weight at birth. For adalimumab, the available data are limited but do not show a worse outcome for pregnancies exposed to this antibody. Nevertheless, the long-term effect of intrauterine exposure to these immunosuppressive agents on the development of the newborn's immune system remains unknown [6]. The ECCO Consensus recommends stopping anti-TNF therapy in the last trimester [30]. However, Nguyen et al in the Toronto Consensus recommend maintaining antiTNF therapy during all trimesters [35]. Therefore, the decision to maintain or stop the drug should be balanced against the risk of relapse, and should be individualized and adapted to the patient's preference. Importantly, the offspring of the PIANO study participants who were exposed to biologics did not have any development delay compared to unexposed babies [29]. On the other hand, combination therapy with an anti-TNF and thiopurine may be associated with preterm birth (OR 2.4, 95\%CI 1.3-4.3) and any pregnancy complication (OR 1.7,95\%CI 1.0-2.2) [26,29]. Preliminary available data concerning the safety of vedolizumab during pregnancy are still limited [26]. Very limited data have been published regarding the safety of ustekinumab in Crohn's disease [36]. Corticosteroids can be used in disease flares, but should be limited to the lowest effective dose and the shortest duration to prevent complications such as gestational diabetes, hypertension, and low birth weight. Although it was reported that corticosteroid use in the first trimester was associated with an increased risk of orofacial malformations, a recent population-based study showed no increased risk [6,37]. Finally, metronidazole is associated with orofacial clefts and ciprofloxacin interferes with intrauterine growth. If an antibiotic is needed, amoxicillin-clavulanic acid is the preferred choice during pregnancy $[6,30]$. The decision to stop a medication should be individualized to each patient, taking into account the probability of relapse and the patient's phenotype, and should always be shared with the patient. 
Indications for surgery in pregnant women with IBD do not differ from those in non-pregnant women [30]. There is no elected timing for surgery, as it depends on the severity of disease activity. Surgery is relatively safe in all trimesters, although there are some reports of spontaneous miscarriage in the first trimester $[6,38]$.

\section{Liver diseases}

Liver diseases during pregnancy can be divided into disorders unique to pregnancy, those coincidental with pregnancy, and pre-existing liver diseases exacerbated by pregnancy [39]. We will focus our discussion on the differential diagnosis of diseases unique to pregnancy (Fig. 1), with a brief note regarding hepatitis E virus (HEV) infection.

\section{Intrahepatic cholestasis of pregnancy (ICP)}

ICP is a cholestatic benign liver disorder of late pregnancy characterized by pruritus and elevation of bile acid levels
( $>10 \mu \mathrm{mol} / \mathrm{L}$ ) and serum aminotransferases. It is a reversible type of hormonally influenced cholestasis and spontaneous relief of symptoms usually occurs within 2-3 weeks after delivery $[40,41,42]$. The time needed for normalization of liver tests may be longer, up to 6-8 weeks. ICP tends to occur in older, multiparous women and twin gestations. A previous history of ICP, cholestasis during oral contraceptive therapy, and family history of both ICP and contraceptive-induced cholestasis are associated with an increased risk of ICP [40].

The pathophysiology is linked to the hormonal status and genetic predisposition of the patient. Higher levels of estrogen and progesterone induce cholestasis by a lower secretion of bile acids. Estrogen decreases the permeability of the basolateral membrane of hepatocytes, whereas progesterone induces this event by inhibiting glucuronosyltransferase. Recent studies have investigated the hepatobiliary transporter genes. Mutations in the hepatic phospholipid transporters (MDRD3, ABCB4), and bile salt export pump (BSEP, ABCB11) have been reported in pregnant women with ICP [40].

The diagnosis is based on the presence of pruritus that starts in the palms and soles and usually worsens in the evening. Jaundice can be developed within 1-4 weeks after

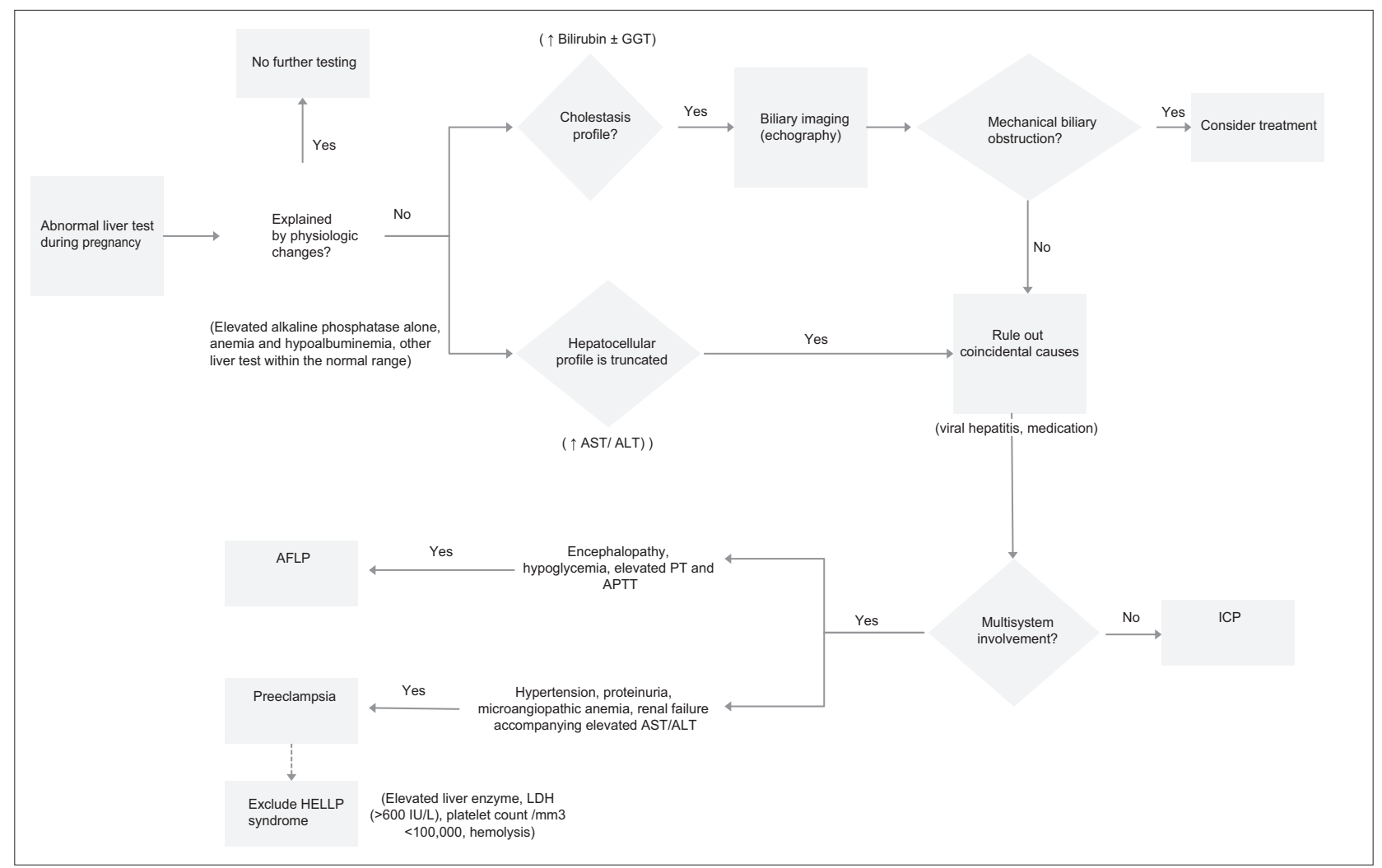

Figure 1 Approach to abnormal liver test during pregnancy. Firstly, exclude anomalies otherwise explained by physiologic changes during pregnancy (elevation of alkaline phosphatase). Then, for a cholestasis profile, mechanical causes of biliary obstruction need to be excluded. If imaging exams are within normal or the patient has a hepatocellular profile, rule out coincidental causes (medication, viral hepatitis). For multisystem involvement in liver diseases unique to pregnancy, consider AFLP (signs of hepatic dysfunction) or preeclampsia. Exclude HELLP syndrome. Pruritus without multisystem involvement leads to ICP

AFLP, acute fatty liver of pregnancy; HELLP, hemolysis, elevated liver enzymes and low platelet count; ICP, intrahepatic cholestasis of pregnancy; $A S T$, aspartate aminotransferase; ALT, alanine aminotransferase; GGT, $\gamma$-glutamyl transferase; $L D H$, lactate dehydrogenase; $P T$, prothrombin time; APTT, activated partial thromboplastin time 
the onset of the pruritus. Steatorrhea can be present and fat malabsorption can lead to vitamin $\mathrm{K}$ deficiency, resulting in prolonged PT and postpartum hemorrhage [40]. Laboratory findings are characterized by an increase in total bile acid levels ( $>12 \mathrm{mmol} / \mathrm{L}$ ) and a 2-10 fold increase in aminotransferases, exceptionally reaching $1000 \mathrm{UI} / \mathrm{L}$. GGT elevation is present in $1 / 3$ of the cases. If GGT is elevated, the patient is more likely to have a genetic component of liver disease [43]. AP may also rise up to 4-fold, but this is not helpful for the diagnosis, since AP is elevated in pregnancy because of placenta production. Hyperbilirubinemia rarely reaches $6 \mathrm{mg} / \mathrm{dL}$ and is found in one quarter of patients. Although ICP is considered a benign disorder to the mother, it is associated with an increased risk of preterm delivery, meconium staining of amniotic fluid, fetal bradycardia, fetal distress and fetal death. The pathogenesis of these fetal outcomes is not completely understood, but it is postulated that higher concentrations of bile acids may be toxic and lead to sudden development of fetal arrhythmia or vasospasm of the placental chorionic surface vessels [41].

The therapeutic approach to ICP includes relief of maternal symptoms and obstetric management to prevent fetal distress. Ursodeoxycholic acid (UDCA) is one of the preferred agents in ICP. The mechanism of UDCA is unknown. A daily dose ranging from $10-15 \mathrm{mg} / \mathrm{kg}$ is effective at reducing pruritus, decreasing the total serum bile acid, ALT and bilirubin levels, allowing delivery closer to term [44]. Early delivery is the only option that really improves clinical outcome. General agreement suggests that delivery should not be delayed after week 37 of gestation [42].

\section{Preeclampsia/eclampsia}

Preeclampsia is defined by the presence of hypertension and proteinuria after the $20^{\text {th }}$ week of gestation; it affects $2-8 \%$ of all pregnancies. Eclampsia is considered when pregnant women with preeclampsia develop seizures without any other explanation. Preeclampsia/eclampsia is a multisystemic disorder characterized by an abnormal vascular response to placenta growth associated with vasoconstriction, endothelial dysfunction, metabolic changes and increased inflammatory responses $[40,45]$. Hepatic involvement may be explained by hepatic arterial vasospasm and precipitation of fibrin within the portal and periportal areas of the liver lobule, which may result in lobular ischemia and hepatocyte necrosis.

The diagnosis is based on hypertension with blood pressure above $140 / 90 \mathrm{mmHg}$ in two consecutive measures (interval $6 \mathrm{~h}$ ) and proteinuria: urine proteins $\geq 0.3 \mathrm{~g}$ in a 24 -h urine specimen, a protein $(\mathrm{mg} / \mathrm{dL}) /$ creatinine $(\mathrm{mg} / \mathrm{dL})$ ratio of 0.3 or higher, or a urine dipstick protein of 1 [40]. Women complain of persistent and severe headache with vomiting, peripheral edema, diplopia and blurred vision. Abnormal live enzymes are present in $20 \%$ to $30 \%$ of cases, with mild to moderate elevation (1.5-5 times normal). Conjugated bilirubin, albumin and PT tend to remain normal [47]. Preeclampsia is considered as severe if the following features are present: pulmonary edema, seizures, oliguria, thrombocytopenia $(<100,000 / \mu \mathrm{L})$, abnormal liver enzymes associated with persistent epigastric or right upper-quadrant pain and central nervous system symptoms such as headaches, blurred vision, blindness or altered mental status [44]. Preterm delivery (15-65\%) and fetal growth restriction (10-25\%) are the most common adverse consequences to the fetus [45].

The liver condition associated with preeclampsia/eclampsia does not need specific treatment and the medical approach is based on blood pressure control, relief of associated symptoms and prompt treatment of seizures [40]. After 37 weeks, women with preeclampsia should be delivered [46]. In preeclampsia cases with severity criteria, delivery should be considered at week 34 or even before (24-34 weeks), if there is fetal or maternal worsening.

\section{Hemolysis, elevated liver enzymes, and low platelet count (HELLP) syndrome}

Although the idea is controversial, some propose that HELLP is a severe form of preeclampsia. Others believe that it is an entity of is own [47]. HELLP syndrome is defined by the presence of hemolysis, elevated liver enzymes and thrombocytopenia. It occurs in $0.17-0.85 \%$ of all pregnancies, more frequently in older multiparous Caucasian women ( $>34$ years). Seventy percent of cases are diagnosed antepartum, most frequently between the $27^{\text {th }}$ and $37^{\text {th }}$ week of gestation [48]. The other $30 \%$ are diagnosed after delivery. Of these, $90 \%$ had preeclampsia during pregnancy. Approximately $5-10 \%$ of women with severe preeclampsia develop HELLP. The pathogenesis seems to be similar to preeclampsia, with generalized endothelial and microvascular injury resulting in microangiopathic anemia, periportal hepatic necrosis and thrombocytopenia [40].

Most of the patients complain of headache, nausea and vomiting, visual disorders and epigastric pain, and physical examination reveals pain in the right upper abdominal quadrant, weight gain and generalized edema. Early laboratory findings include hemolysis, characterized by high levels of lactate dehydrogenase (>600 UI/L) with decreased haptoglobin values, non-conjugated hyperbilirubinemia, elevated aminotransferases and thrombocytopenia.

The Mississippi Classification was developed to categorize the severity of HELLP syndrome (Table 3 ). If the platelet count is lower than $50,000 / \mathrm{mm}^{3}$, an association with disseminated intravascular coagulation can occur. The lack of an increase in platelet levels $96 \mathrm{~h}$ from delivery indicates a worse prognosis with possible evolution to multiple organ failure [49]. PT and activated partial thromboplastin time (APTT) are normal in the early stages. As a consequence of secondary fibrinolysis and platelet aggregation, levels of fibrin products, d-dimers and thrombin-antithrombin complexes may be high. Some patients have renal involvement.

Acute liver failure is a rare complication with an incidence of 5/100,000. Spontaneous hepatic rupture occurs in less than $2 \%$ of cases. Affected patients usually present with right upper quadrant abdominal pain accompanied by nausea, 
Table 3 Mississippi classification of HELLP syndrome

\begin{tabular}{llll}
\hline HELLP class & Platelet count & AST/ALT & LDH \\
\hline 1 & $\leq 50,000 / \mathrm{L}$ & $\geq 70 \mathrm{IU} / \mathrm{L}$ & $\geq 600 \mathrm{IU} / \mathrm{L}$ \\
2 & $50,000-100,000 / \mathrm{L}$ & & \\
3 & $100,000-150,000 / \mathrm{L}$ & $\geq 40 \mathrm{IU} / \mathrm{L}$ & \\
\hline
\end{tabular}

Patients with severe disease are included in class 1 , characterized by a low platelet count $\leq 50,000 / \mathrm{L}$ and AST/ALT $\geq 70$ IU/L Platelets between 50,000 and 100,000 help define class 2 patients. Those with mild thrombocytopenia ( $>100,000 / \mathrm{L}$ ), with mild elevations of AST and ALT are class 3 . $\mathrm{LDH}$ is $\geq 600 \mathrm{IU} / \mathrm{L}$ in every class.

HELLP, hemolysis, elevated liver enzymes and low platelet count; AST, aspartate aminotransferase; $A L T$, alanine aminotransferase; $L D H$, lactate dehydrogenase

vomiting, hypertension or hypovolemic shock, as a result of Glisson's capsule rupture [50]. Ultrasound or magnetic resonance imaging usually make the diagnosis of subcapsular or intraparenchymal hemorrhage and hepatic rupture [40]. Management includes both emergent fetus extraction and hepatic surgery. Liver transplant may be needed when fulminant hepatic failure or uncontrolled hemorrhage are present during laparotomy [50].

Perinatal mortality is usually caused by premature detachment of the placenta with intrauterine asphyxia. Maternal mortality is $1-3.5 \%$ as a result of coagulation and hemorrhagic disorders [49].

The management of these patients should be performed in Intensive Care Units and includes dialysis and ventilator support in severe cases, antithrombotic agents, aspirin in low doses, prostacyclin, immunosuppressive agents, steroids and fresh frozen plasma. However, delivery is the only specific therapy in HELLP syndrome [40].

\section{Acute fatty liver of pregnancy (ALFP)}

ALFP is a rare but potential serious disorder that occurs in the third trimester of pregnancy (between the $30^{\text {th }}$ and $38^{\text {th }}$ weeks) or early postpartum [40]. Nowadays, maternal and perinatal mortality are approximately $18 \%$ and $25 \%$ respectively. AFLP may result from mitochondrial dysfunction in the fetal liver. Long-chain 3-hydroxyacyl-CoA dehydrogenase is one of the most important enzyme deficits documented. Deficiency in this enzyme leads to the accumulation of long-chain fatty acids in fetal and maternal circulation. As the mother is heterozygote for the enzyme, long-chain fatty-acid metabolism is impaired and becomes toxic with microvesicular fatty infiltration of the hepatocytes.

Patients often present with nonspecific symptoms, such as anorexia, nausea, vomiting, headache and abdominal pain. The most important physical findings are fever and jaundice, which occurs in $70 \%$ of patients. Approximately 50\% of patients have signs of preeclampsia [40]. Analytic findings include an elevated white blood cell count $(>15,000 / \mathrm{L})$ accompanied by normal hematocrit, unless hemorrhage or hemolysis is also present. PT and APTT are both prolonged, with low levels of fibrinogen. Aminotransferases are elevated, with levels increased up to 300-500 UI/L, and can be associated with lactic acidosis, hyperbilirubinemia and hypoglycemia.

AFLP is a differential diagnosis of preeclampsia, HELLP syndrome, ICP, and viral hepatitis. The tendency towards hypoglycemia, hyperammonemia and the prolongation of PT and APTT help distinguish AFLP from HELLP [39]. Hepatic serology should also be part of the complementary exams.

AFLP is a medical and obstetrical emergency. Prompt delivery and intensive supportive care are the cornerstones in the management of AFLP [49].

\section{HEV infection}

$\mathrm{HEV}$ is an enterically transmitted virus that causes clinically apparent hepatitis in developing countries as India, Asia, Africa and Central America. The main source of transmission of $\mathrm{HEV}$ is contaminated drinking water. Recently, an increasing number of cases have emerged in developed countries as a result of zoonotic infections [51]. One of the features that distinguish HEV from the other hepatotropic viruses is its virulent course during pregnancy, with more frequent progression to fulminant hepatic failure. Mortality is up to $10-$ $20 \%$ in pregnant women. Acute HEV infection is especially severe during the $2^{\text {nd }}$ and $3^{\text {rd }}$ trimesters [52].

It has been postulated that higher mortality is associated with hormonal and immunological changes. Navaneethan et al reported that there is a clear shift from Th1 to Th2 cell paradigm during pregnancy and the levels of most cytokines are decreased, especially during the initial 20 weeks of pregnancy [51]. In addition, higher levels of progesterone, estrogen and $\beta$-HCG have been associated in animal studies with a suppressive effect in cell-mediated immunity. Both these mechanisms can lead to higher susceptibility to HEV virulence [51]. Nevertheless, there is no study documenting a causal relation between these physiological findings and HEV infection.

Most of the studies of HEV in pregnant women were conducted in high-prevalence countries. Encephalopathy was the most common complication of acute infection during pregnancy, followed by gastrointestinal bleeding, renal failure, and seizures. Pregnant women with acute liver failure have a worse prognosis than those who just have acute hepatitis, with higher rates of intrauterine death $(78.6 \%$ vs. $11.4 \%)$, preterm delivery $(65.7 \%$ vs. $17.3 \%)$ and maternal mortality ( $56.2 \%$ vs. $0.9 \%)$ [53]. Vertical transmission has also been reported and can occur at any point during pregnancy. However, HEV infection is usually self-limited in the neonate and fulminant hepatitis is rare.

Although HEV infection may be severe during pregnancy, treatment is only supportive. Transplantation can be considered in selected cases [54]. The best approach is through prevention. Two different recombinant vaccines have been developed, but are not yet approved.

\section{Endoscopy in pregnancy}

Gastrointestinal endoscopy is usually considered a safe procedure. However, many potential risks are associated 
with endoscopy during pregnancy [55]. A recent Swedish population-based cohort study found endoscopy to be associated with an increased risk of preterm birth or small size for gestational age, even though no association with congenital malformation or stillbirth was found [56].

The most common indications for upper endoscopy in pregnant women are major or continued gastrointestinal hemorrhage, dysphagia and refractory nausea and vomiting. The most common cause of non-variceal acute hemorrhage is a Mallory-Weiss tear. Women who have esophageal varices or severe liver disease should be advised about the risk of variceal rupture. Endoscopy is usually not indicated in severe nausea and vomiting during pregnancy or HG, but if the patient has severe nausea and vomiting, accompanied by abdominal pain refractory to medical treatment, endoscopy may be appropriate for the diagnosis of major peptic ulcers or gastric outlet obstruction. Epinephrine injection, thermocoagulation and electrocoagulation have proved successful in all patients during pregnancy, excluding those who needed surgery. Epinephrine can decrease blood flow to the fetus. However, no adverse effects have been reported in the available literature [34,57]. In electrocoagulation, amniotic fluid may conduct electric current to the fetus, so a grounding pad should be placed away from the uterus and bipolar electrocautery should be used to minimize the risk. However, there are limited data concerning hemostasis for non-variceal bleeding in pregnant patients and the therapeutic technique is based on expert opinion from non-pregnant patients. Sclerotherapy and endoscopic band ligation are safe procedures during pregnancy [55].

Sigmoidoscopy and colonoscopy are indicated in the evaluation of major lower gastrointestinal bleeding, suspicion of colonic mass and severe persistent diarrhea with unknown etiology [55]. Sigmoidoscopy is considered to be safe and effective when obstetrical consultation is performed. There are limited data evaluating the safety and adverse events of colonoscopy during pregnancy, so this examination must only be performed when strictly necessary. However, when required before surgery, colonoscopy should be performed to assess a suspected colon cancer/mass, uncontrolled severe hemorrhage, colonic stricture of unknown cause, or pseudoobstruction. All the elective indications should be deferred until after delivery.

Pregnancy is associated with an increased risk of gallstones. Complications of common bile duct or accessory duct obstruction can occur, including cholecystitis, cholelithiasis and pancreatitis, although these events are rare. There is controversy about the safety of endoscopic retrograde cholangiopancreatography (ERCP) during pregnancy, and data are limited because of the radiation exposure to the fetus and the risk of the procedure in terms of the maternal outcome [30]. Indications for ERCP include recurrent biliary colic, abnormal liver function tests and a dilated bile duct on ultrasonography [58]. Common bile duct stones, biliary pancreatitis and cholangitis are also indications for ERCP during pregnancy. Endoscopic ultrasonography is not contraindicated in pregnancy and may reduce unnecessary interventions in patients who have lower or moderate probability of choledocholithiasis [55].

Sedative drugs may be used safely during pregnancy. Two studies have confirmed that meperidine is not teratogenic and can be used during this period. When sedation with meperidine alone is insufficient, midazolam can be used as adjuvant. However, benzodiazepines are usually not recommended during pregnancy. Diazepam is associated with cleft palate and neurobehavioral disorders. Propofol seems to be a safer option compared to benzodiazepines, as no animal or human teratogenic effects have been reported [59]. Both propofol and midazolam should be avoided during the first trimester. Fentanyl should not be prescribed [60].

\section{Concluding remarks}

The medical approach to the pregnant women is challenging. Clinicians have to evaluate the possibility of unique diseases during pregnancy and to choose the best therapeutic strategy, keeping in mind options that are harmless for the fetus. In this review we have summarized the essential diagnosis and management that should be considered by gastroenterologists when approaching a pregnant woman, in order to improve our clinical guidance. Specific considerations are necessary concerning IBD and endoscopic exams during this period. We also emphasized the need for a multidisciplinary approach in order to prevent pregnancy complications and to define the best strategy and timing for delivery.

\section{References}

1. Ahmed KT, Almashhrawi AA, Rahman RN, Hammoud GM, Ibdah JA. Liver diseases in pregnancy: diseases unique to pregnancy. World J Gastroenterol 2013;19:7639-7646.

2. Tan EK, Tan EL. Alterations in physiology and anatomy during pregnancy. Best Pract Res Clin Obstet Gynaecol 2013;27:791-802.

3. Augustin G, Majerovic M. Non-obstetrical acute abdomen during pregnancy. Eur J Obstet Gynecol Reprod Biol 2007;131:4-12.

4. Body C, Christie JA. Gastrointestinal diseases in pregnancy. Nausea, vomiting, hyperemesis gravidarum, gastroesophageal reflux disease, constipation, and diarrhea. Gastroenterol Clin North Am 2016;45:267-283.

5. Matthews A, Hass DM, O’Mathúna DP, Dowswell T. Interventions for nausea and vomiting in early pregnancy. Cochrane Database Syst Rev 2015; 8:CD007575.

6. van der Woude CJ, Metselaar HJ, Danese S. Management of gastrointestinal and liver diseases during pregnancy. Gut 2014;63:1014-1023.

7. Giacosa A, Morazzoni P, Bombardelli E, Riva A, Porro GB, Rondanelli M. Can nausea and vomiting be treated with ginger extract? Eur Rev Med Pharmacol Sci 2015;19:1291-1296.

8. Pasternak B, Svanström H, Hviid A. Ondansetron in pregnancy and risk of adverse fetal outcomes. N Engl J Med 2013;368:814-823.

9. Shehmar M, MacLean MA, Nelson-Piercy C, Gadsby R, O'Hara M. The management of nausea and vomiting of pregnancy and hyperemesis gravidarum. Royal College of Obstetricians and Gynaecologists Green-top Guideline No. 69, 2016. 
10. Ali RA, Egan LJ. Gastroesophageal reflux disease in pregnancy. Best Pract Res Clin Gastroenterol 2007;21:793-806.

11. Ching C, Lam S. Antacids: indications and limitations. Drugs 1994;47:305-317.

12. Tytgat GN, Heading RC, Müller-Lissner S, et al. Contemporary understanding and management of reflux and constipation in the general population and pregnancy: a consensus meeting. Aliment Pharmacol Ther 2003;18:291-301.

13. Richter JE. Review article: the management of heartburn in pregnancy. Aliment Pharmacol Ther 2005;22:749-757.

14. Gill SK, O'Brien L, Einarson TR, Koren G. The safety of proton pump inhibitors (PPIs) in pregnancy: a meta-analysis. Am J Gastroenterol 2009;104:1541-1545; quiz 1540, 1546.

15. Mahadevan U. Gastrointestinal medications in pregnancy. Best Pract Res Clin Gastroenterol 2007;21:849-877.

16. Pasternak B, Hviid A. Use of proton-pump inhibitors in early pregnancy and the risk of birth defects. $N$ Engl $J$ Med 2010;363:2114-2123.

17. Derbyshire E, Davies J, Costarelli V, Dettmar P. Diet, physical inactivity and the prevalence of constipation throughout and after pregnancy. Matern Child Nutr 2006;2:127-134.

18. Cullen G, O'Donoghue D. Constipation and pregnancy. Best Pract Res Clin Gastroenterol 2007;21:807-818.

19. Wald A. Constipation, diarrhoea and symptomatic hemorrhoids during pregnancy. Gastroenterol Clin North Am 2003;32:309-322.

20. Riis L, Vind I, Politi P, et al; European Collaborative study group on Inflammatory Bowel Disease. Does pregnancy change the disease course? A study in a European cohort of patients with inflammatory bowel disease. Am J Gastroenterol 2006;101:1539-1545.

21. Bharucha AE, Dorn SD, Lembo A, Pressman A; American Gastroenterological Association. American Gastroenterological Association medical position statement on constipation. Gastroenterology 2013;144:211-217.

22. Bailit J. Hyperemesis gravidarium: epidemiologic findings from a large cohort. Am J Obs Gynecol 2005;193:811-814.

23. Ismail SK, Kenny L. Review on hyperemesis gravidarum. Best Pract Res Clin Gastroenterol 2007;21:755-769.

24. Fell DB, Dodds L, Joseph KS, Allen VM, B. B. Risk factors for hyperemesis gravidarum requiring hospital admission during pregnancy. Obstet Gynecol 2006;107(2 Pt 1):277-284.

25. Lagiou PN, Tamimi R, Mucc LA, Trichopoulos D, H.O. A, Hsieh C. Nausea and vomiting in pregnancy in relation to prolactin, estrogens, and progesterone: a prospective study. Obstet Gynecol 2003;101:639-644.

26. McConnell RA, Mahadevan U. Pregnancy and the patient with inflammatory bowel disease: fertility, treatment, delivery, and complications. Gastroenterol Clin North Am 2016;45:285-301.

27. Cornish J, Tan E, Teare J, et al. A meta-analysis on the influence of inflammatory bowel disease on pregnancy. Gut 2007;56:830-837.

28. Mahadevan U, Sandborn WJ, Li DK, Hakimian S, Kane S, Corley DA. Pregnancy outcomes in women with inflammatory bowel disease: a large community-based study from Northern California. Gastroenterology 2007;133:1106-1112.

29. Mahadevan U, Martin CF, Sandler RS, et al. PIANO: A 1000 patient prospective registry of pregnancy outcomes in women with IBD exposed to immunomodulators and biologic therapy. Gastroenterology 2012;142 Suppl 1:S-149.

30. Van der Woude CJ, Ardizzone S, Bengtson MB, et al. The second European evidenced-based consensus on reproduction and pregnancy in inflammatory bowel disease. J Crohns Colitis 2015;9:107-124.

31. Bálint A, Berényi A, Farkas K, et al. Pregnancy does not affect fecal calprotectin concentration in healthy women. Turk J Gastroenterol 2017;28:171-175.

32. Julsgaard M, Hvas CL, Gearry RB, et al. Fecal calprotectin is not affected by pregnancy: clinical implications for the management of pregnant patients with inflammatory bowel disease. Inflamm Bowel Dis 2017;23:1240-1246.

33. Meuwis M, Vernier-Massouille G, Grimaud J, et al. Serum calprotectin as a biomarker for Crohn's disease. J Crohns Colitis 2013;7:e678-e683.

34. Qureshi WA, Rajan E, Adler DG, et al; American Society for Gastrointestinal Endoscopy. ASGE Guideline: Guidelines for endoscopy in pregnant and lactating women. Gastrointest Endosc 2005;61:357-362.

35. Nguyen GC, Seow CH, Maxwell C, et al; Canadian Association of Gastroenterology. The Toronto consensus statements for the management of inflammatory bowel disease in pregnancy. Gastroenterology 2016;150:734-757.

36. Levy RA, de Jesús GR, de Jesús NR, Klumb EM. Critical review of the current recommendations for the treatment of systemic inflammatory rheumatic diseases during pregnancy and lactation. Autoimmun Rev 2016;15:955-963.

37. Park-Wyllie L, Mazzotta P, Pastuszak A, et al. Birth defects after maternal exposure to corticosteroids: prospective cohort study and meta-analysis of epidemiological studies. Teratology 2000;62:385-392.

38. Hill J, Clark A, Scott NA. Surgical treatment of acute manifestations of Crohn's disease during pregnancy. J R Soc Med 1997;90:64-66.

39. Schutt VA, Minuk GY. Liver diseases unique to pregnancy. Best Pract Res Clin Gastroenterol 2007;21:771-792.

40. Pusl T, Beuers U. Intrahepatic cholestasis of pregnancy. Orphanet $J$ Rare Dis 2007;2:26.

41. Ozkan S, Ceylan Y, Ozkan OV, Yildirim S. Review of a challenging clinical issue: Intrahepatic cholestasis of pregnancy. World $J$ Gastroenterol 2015;21:7134-7141.

42. Lammert F, Marschall HU, Glantz A, Matern S. Intrahepatic cholestasis of pregnancy: molecular pathogenesis, diagnosis and management. J Hepatol 2000;33:1012-1021.

43. Glantz A, Marschall HU, Lammert F, Mattsson LA. Intrahepatic cholestasis of pregnancy: a randomized controlled trial comparing dexamethasone and ursodeoxycholic acid. Hepatology 2005;42:1399-1405.

44. Sibai B, Dekker G, Kupferminc M. Pre-eclampsia. Lancet 2005;365:785-799.

45. Tran TT, Ahn J, Reau NS. ACG Clinical Guideline: liver disease and pregnancy. Am J Gastroenterol 2016;111:176-194; quiz 196.

46. Rahman TM, Wendon J. Severe hepatic dysfunction in pregnancy. QJM 2002;95:343-357.

47. Mihu D, Costin N, Mihu CM, Seicean A, Ciortea R. HELLP syndrome - A multisystemic disorder. J Gastrointestin Liver Dis 2007; 16:419-424.

48. O'Brien J, Buckley O, Munk P, Torreggiani W. An unusual case of elevated liver enzymes: hepatic necrosis following HELLP syndrome. Eur Radiol 2007;17:289-291.

49. Pavlis T, Aloizos S, Aravosita P, et al. Diagnosis and surgical management of spontaneous hepatic rupture associated with HELLP syndrome. J Surg Educ 2009;66:163-167.

50. Navaneethan U, Al Mohajer M, Shata MT. Hepatitis E and pregnancy: understanding the pathogenesis. Liver Int 2008;28:1190-1199.

51. Shinde N, Patil T, Deshpande A, Gulhane R, Patil M, Bansod Y. Clinical profile, maternal and fetal outcomes of acute hepatitis e in pregnancy. Ann Med Health Sci Res 2014;4:S133-S139.

52. Rac MW, Sheffield JS. Prevention and management of viral hepatitis in pregnancy. Obstet Gynecol Clin North Am 2014;41:573-592.

53. Shalimar, Acharya SK. Hepatitis E and acute liver failure in pregnancy. J Clin Exp Hepatol 2013;3:213-224.

54. Savas N. Gastrointestinal endoscopy in pregnancy. World J Gastroenterol 2014;20:15241-15252. 
55. Ludvigsson JF, Lebwohl B, Ekbom A, et al. Outcomes of pregnancies for women undergoing endoscopy while they were pregnant: a nationwide cohort study. Gastroenterology 2017;152:554-563.

56. O'Mahony S. Endoscopy in pregnancy. Best Pract Res Clin Gastroenterol 2007;21:893-899.

57. Tang SJ, Mayo MJ, Rodriguez-Frias E, et al. Safety and utility of
ERCP during pregnancy. Gastrointest Endosc 2009;69:453-461.

58. Neuman G, Koren G. Safety of procedural sedation in pregnancy. J Obstet Gynaecol Can 2013;35:168-173.

59. Shergill AK, Ben-Menachem T, Chandrasekhara V, et al; ASGE Standard of Practice Committee. Guidelines for endoscopy in pregnant and lactating women. Gastrointest Endosc 2012;76:18-24. 